\title{
Adaptive Sliding Mode Control of MEMS AC Voltage Reference Source
}

\author{
Ehsan Ranjbar, Ali Mehrnezhad, and Amir Abolfazl Suratgar \\ Department of Electrical Engineering, Amirkabir University of Technology (AUT), 424 Hafez Ave., Tehran, Iran
}

Correspondence should be addressed to Amir Abolfazl Suratgar; a-suratgar@aut.ac.ir

Received 5 May 2017; Accepted 6 July 2017; Published 20 September 2017

Academic Editor: Guimin Chen

Copyright (C) 2017 Ehsan Ranjbar et al. This is an open access article distributed under the Creative Commons Attribution License, which permits unrestricted use, distribution, and reproduction in any medium, provided the original work is properly cited.

The accuracy of physical parameters of a tunable MEMS capacitor, as the major part of MEMS AC voltage reference, is of great importance to achieve an accurate output voltage free of the malfunctioning noise and disturbance. Even though strenuous endeavors are made to fabricate MEMS tunable capacitors with desiderated accurate physical characteristics and ameliorate exactness of physical parameters' values, parametric uncertainties ineluctably emerge in fabrication process attributable to imperfections in micromachining process. First off, this paper considers applying an adaptive sliding mode controller design in the MEMS AC voltage reference source so that it is capable of giving off a well-regulated output voltage in defiance of jumbling parametric uncertainties in the plant dynamics and also aggravating external disturbance imposed on the system. Secondly, it puts an investigatory comparison with the designed model reference adaptive controller and the pole-placement state feedback one into one's prospective. Not only does the tuned adaptive sliding mode controller show remarkable robustness against slow parameter variation and external disturbance being compared to the pole-placement state feedback one, but also it immensely gets robust against the external disturbance in comparison with the conventional adaptive controller. The simulation results are promising.

\section{Introduction}

Basic principal of AC and DC MEMS-based voltage references was provided by [1] for the first time. They introduced a microsized parallel-moving plate capacitor which is used in a simple circuit to provide a reference voltage. The characteristic of this capacitor which is based on high mechanical stability of single crystalline silicon causes precise output voltage across its plates. Furthermore, low production cost and small size make these voltage references unique devices for electronic applications. After Suhonen, many other researchers started to work on these voltage reference sources to improve their accuracy. Since the fabrication characteristics of MEMS tunable capacitor as the main element of MEMS-based voltage references are very critical, there are several papers which are focused on micromachining process of the MEMS tunable capacitors [2-7]. The main purpose of these works is to propose and fabricate new designs of microsized tunable capacitors and show the improvement of obtained MEMS-based voltage references.
Utilizing tunable capacitor in MEMS-based voltage references is associated with pull-in phenomenon. This property stems from electromechanical coupling between the electrostatic force being applied on the capacitor plates and the spring force. As it is illustrated in Figures 1(a) and 1(b), the electrostatic force intensifies as the electrical charge across the capacitor plates accumulates. Thus, movable plate of capacitor is steered towards the fixed plate gently, until the spring force can no longer withstand the electrostatic force. This point is acquainted as the pull-in point. Near the pull-in point, the voltage alteration across capacitor plates is proportional to the square of deflection in the movable plate. Hence, this capacitor can furnish a regulated voltage while working around the pull-in point. Consequently, the MEMS-based voltage references are exploited near the pull-in point of their MEMS tunable capacitors. According to [8], mechanical stress and electrical charge over dielectric layer are two reasons which cause instability around the pull-in point. They stated that using AC voltage, instead of DC one, decreases the charge aggregation across the dielectric layer. Using $\mathrm{AC}$ voltage 


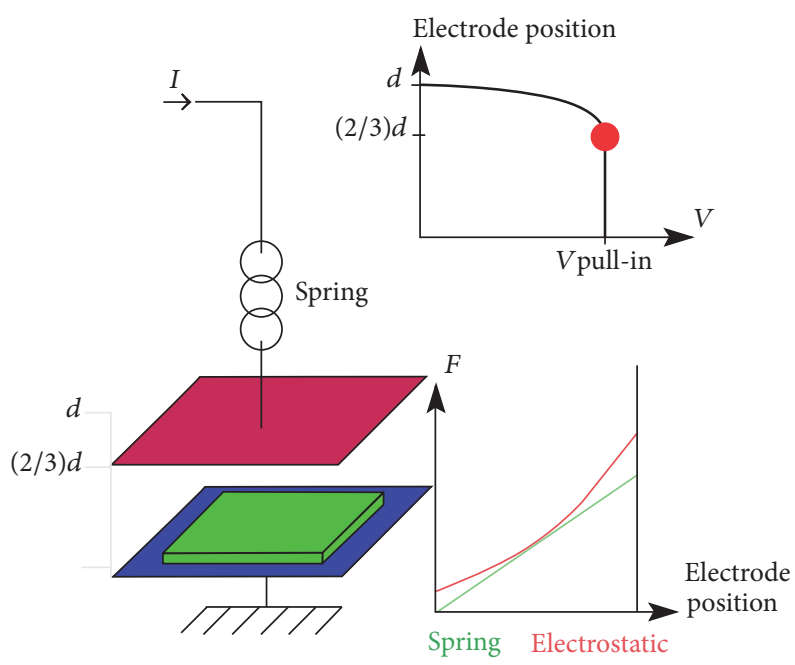

(a)

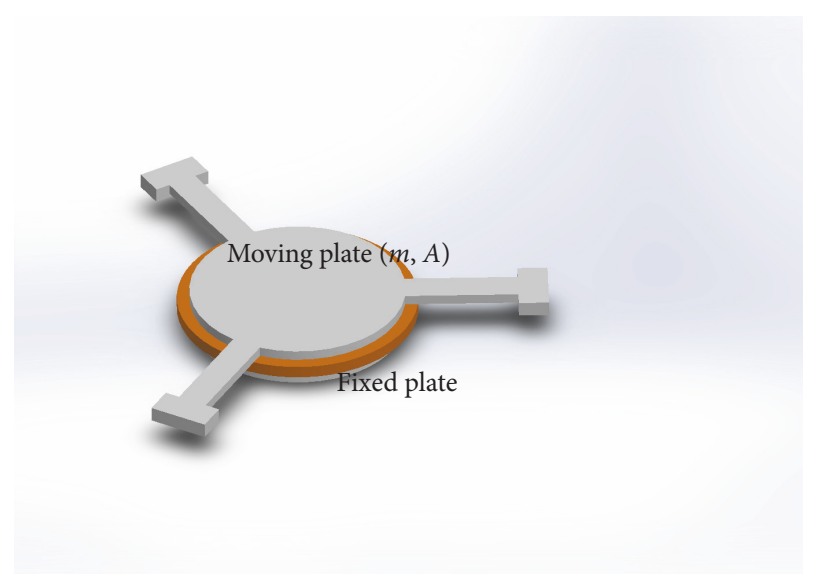

(c)

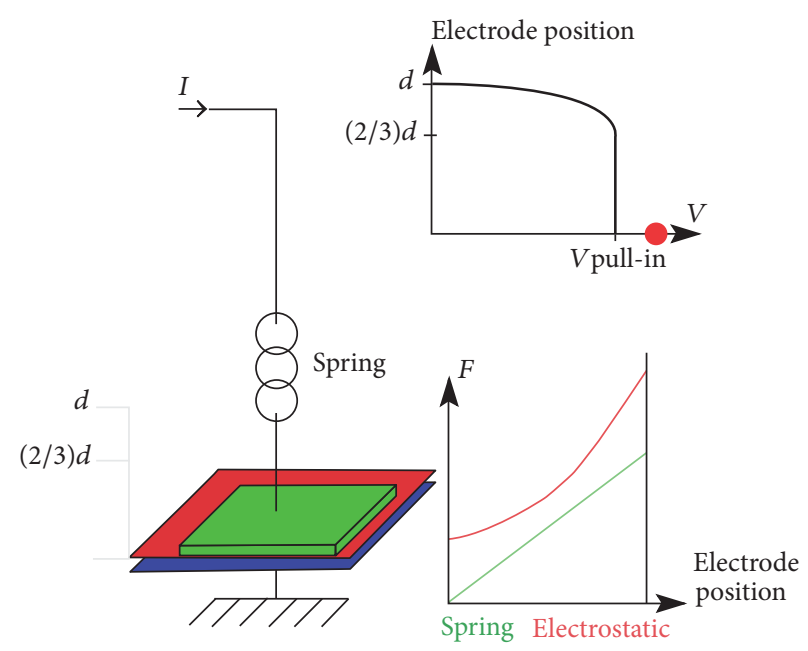

(b)

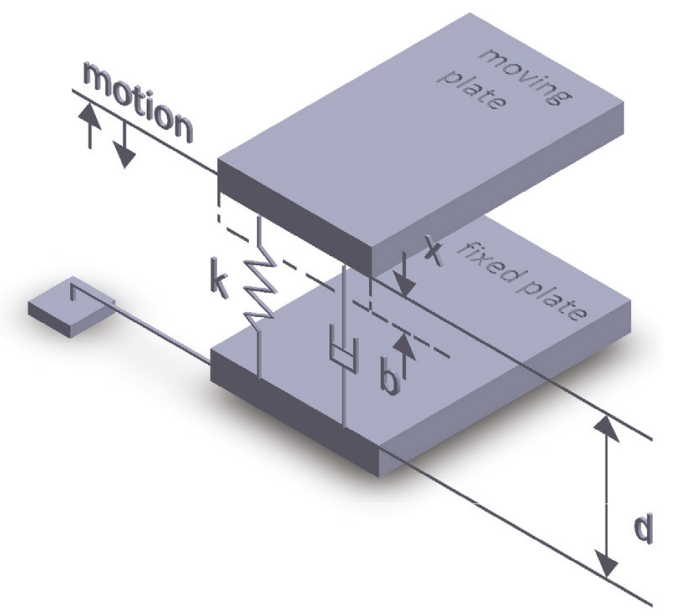

(d)

FIGURE 1: MEMS tunable capacitor; (a) its model and pull-in point: pull-in point transpires when movable electrode reaches one-third of its initial distance to fixed electrode; (b) tunable capacitor mislays its stability beyond pull-in voltage; (c) top plate puts in motion in a piston way; (d) lumped model of the MEMS tunable capacitor.

as the input resulted in appearance of MEMS-based AC voltage reference sources. In this paper, the operation of these MEMS-based AC voltage reference sources is considered. Nowadays, the advanced microfabrication technology helps us to create MEMS tunable capacitors with highly accurate dimensions and geometries and less mechanical stress. Nevertheless, applying controllers to MEMS-based voltage reference sources is necessary because it has great impact on increasing the long term stability and accuracy of reference voltage. It is not blowing it out of proportion that applying various control algorithms to MEMS devices to yield error subside and a better performance is a nonnegligible problematic issue [9-14]. In addition, adaptive and robust control methods lie in the center of meticulousness and challenge due to deficiency in modeling of MEMS devices and the parametric uncertainties in fabrication procedures [1522].
A state space description was proposed in [23] to apply various control algorithms to MEMS tunable capacitors. The contemplation of the controller is to place and maintain the movable plate of the capacitor in the pull-in point while the capacitor's parameters, like spring stiffness, get changed during the time. When the system's parameters are deemed steady, this aim is obtained utilizing a simple pole-placement state feedback controller. Nevertheless, the problem arises when we would know that there are some ineluctable uncertainties in the system parameters. For instance, the exact value of spring stiffness is not known.

This paper considers application of an adaptive sliding mode (ASM) controller design [24] to control the system and attain perfect tracking so that the position trajectory of the movable plate would flawlessly track the desired pull-in trajectory in defiance of jumbling uncertain parameters and also aggravating external disturbance; it is beneficial when 

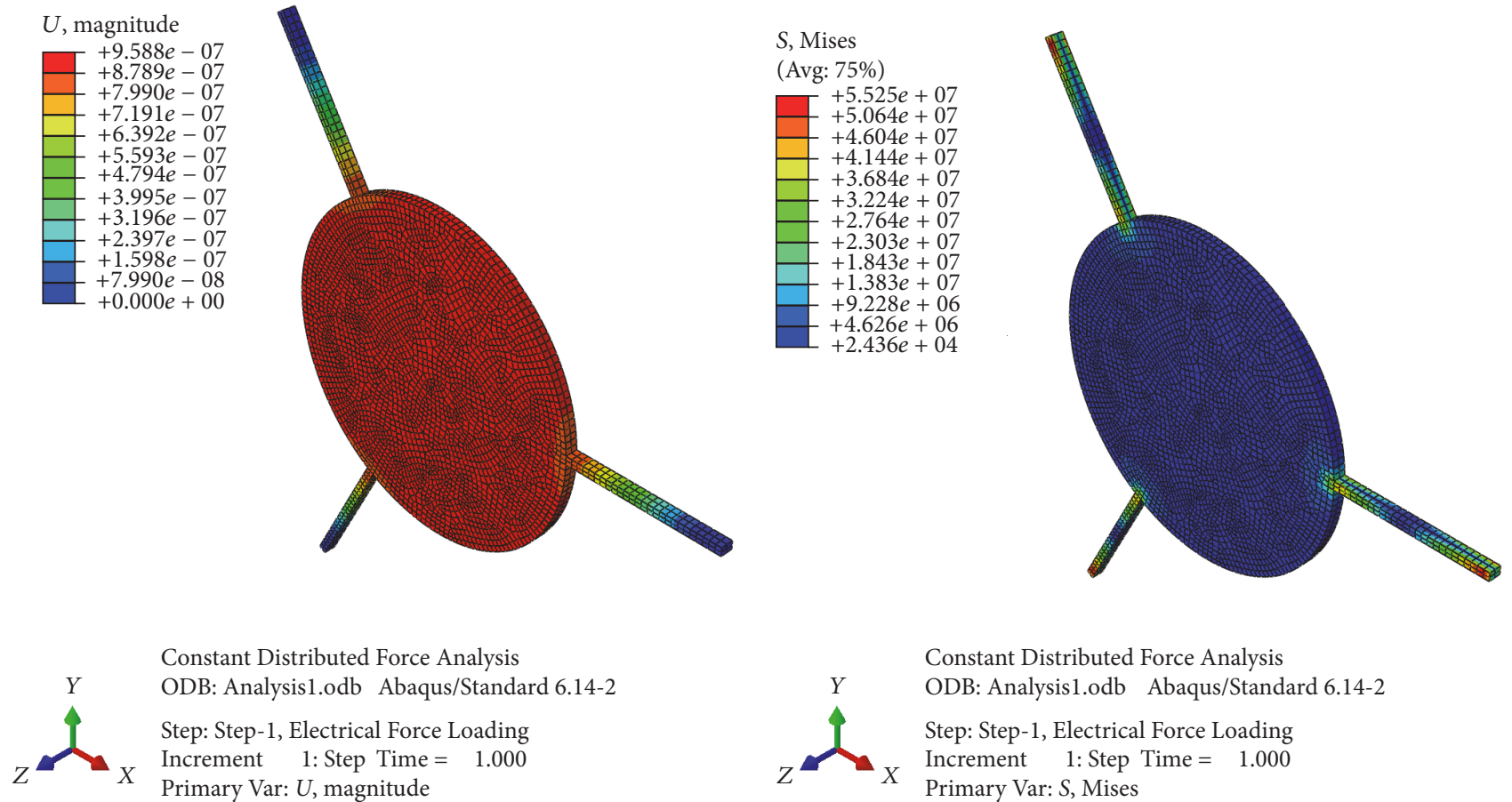

Constant Distributed Force Analysis

ODB: Analysis1.odb Abaqus/Standard 6.14-2

Step: Step-1, Electrical Force Loading

Increment $\quad 1:$ Step Time $=1.000$

Primary Var: $U$, magnitude

Deformed Var: $U$ Deformation Scale Factor: $+1.000 e+00$

(a)

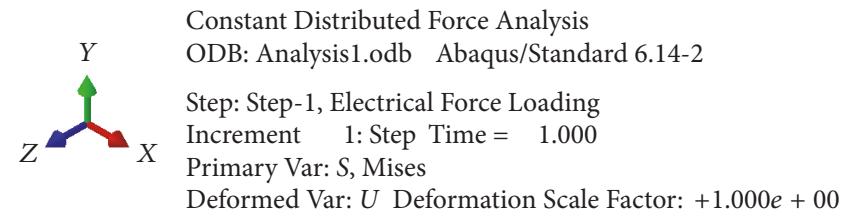

(b)

FIGURE 2: Tension and displacement: (a) displacement of the seeds in top plate; as it is seen, the desired displacement, $67 \mu \mathrm{m}$, is not attained due to simplifying assumptions in modeling; (b) stress distribution on top plate due to the applied electrostatic force.

showing conceded robustness against the disturbance which is imposed on the system from the input channel. It also shows up remarkable robustness against the slow parameter variation; the designed presented scheme is also capable of perfect tracking when the parameters vary slowly. It is worth notifying that the spring stiffness and damping coefficients could alter gradually as the environmental conditions, like temperature, get changed.

\section{Operating Principles}

In this section, operating principles of the MEMS tunable capacitor are investigated and its characteristics are illustrated. A tunable capacitor has a fixed plate and a movable one. In this paper, one of the typical tunable capacitors, depicted on Figures 1(c) and 1(d), is conversed. It is a MEMS parallel plate tunable capacitor and incorporates a movable plate which alters the interplate gap. As it got mentioned in Introduction, the challenging issue, that we are dealing with in this paper, is assuaging the tracking performance quality of the electrically aggregated plate in the tunable capacitor in the presence of parameter uncertainty. It is depicted by simulating the parallel plate in Abaqus software that hardly does the plate, designed according to the structural parameters given in [26], displace so that it would not get maintained in the desiderated pull-in point, as seen in Figure 2. It is conjectured that the simplifying assumption in modeling procedure could be one source of parameter uncertainty in stiffness factor of the suspending beams. One would refer to the depletion of desired mechanical characteristic due to aging and alteration of working condition which consequently results in parameter uncertainty. Unfortunately, defects in fabrication procedure can be another source of slight uncertainty in dominating parameters like stiffness and damping factors. That is why contemplating a control system which would cope with parameter uncertainty could not be deprived of worthwhile challenge and attention.

Capacitance of a MEMS tunable capacitor changes with the gap; it is defined by

$$
C=\frac{\epsilon A}{d-x} .
$$

The parameters in (1), $\epsilon, A, d$, and $x$, consecutively symbolize the permittivity of the medium, the electrode area, the initial gap between plates, and the movable plate deviation. The capacitor is actuated with an AC current defined by

$$
I=I_{m} \sin (\omega t) .
$$

Electrostatic force $F_{E}$ captivates electrodes towards each other whereas spring force, defined by $k x$, attempts to impede plates from appealing to each other. These two forces are balanced through (3) looking at Figure 1(d) [27]. This balance strive is depicted in following equation [26]:

$$
F_{E}=\left|-\frac{\partial E_{I}}{\partial x}\right|=\frac{I_{m}^{2} \cos ^{2}(\omega t)}{2 \epsilon A \omega^{2}}=k x+b \frac{d x}{d t}+m \frac{d^{2} x}{d t^{2}} .
$$


The variables and parameters in (3), $F_{E}, E_{I}, x, k, m$, and $b$, are, respectively, the electrostatic force generated by the AC current, the electrostatic energy, plate distance, spring constant, mass of the movable electrode, and damping coefficient due to existing gas between plates. The actuation current frequency, $\omega / 2 \pi$, is nearly supposed to be much higher than the mechanical resonance frequency defined by $\omega_{0} / 2 \pi=\sqrt{k / m} / 2 \pi$. Moreover, the movement of the movable plate gets damped when the stable position is at the access. The damping coefficient is related to gas damping of the component. The mechanical quality factor of the moving plate capacitor, called $Q$, can be controlled by adjusting the gas pressure inside the component enclosure:

$$
Q=\frac{1}{2 \zeta}
$$

Neglecting the slide film damping with regard to movement constraint, $b$ is charged for squeeze film one in subsequent simulation; however, this effect is handled physically by certain fabrication precautions. For a quantitative analysis the compressibility of the gas needs to be taken into account. The precautionary actions go as follows regarding [26]:

(i) Device is operated at a frequency much higher than the resonance frequency since the gas has enough time to flow away from the gap causing dissipation in low frequencies. Increasing the frequency, the gas film is trapped and squeezed between the electrodes and behaves like a spring with low dissipation.

(ii) The sensor is encapsulated in an inertial protective gas such as $1 \mathrm{~atm}$ nitrogen or inert gases like argon. High gas pressure works as a mechanical damper; the pressure inside the cavity is high enough to overdamp the cantilever movement in such a way that the mechanical $Q$ value is well below 1 .

(iii) Nice sealing packages such as metal packages (TOcan and DIL) and Low Temperature Co-fired Ceramic (LTCC) are applied on the hermetic package not only to hamper gas leakage and pressure fall but also to reduce sensitivity to changes in environmental humidity and pressure.

(iv) Before the final lid seal, a bake is usually performed to drive out any trapped gas or moisture since metal surfaces can be very hydrophilic and any residual chemicals from the manufacturing process may react chemically and cause voltage drift.

(v) An optimizing movable plate mass calculation should be done to minimize molecular vibration of the damping gas and the consequent mechanical noise of the component. The molecules in any substance at a temperature above absolute zero are permanently vibrating which causes arbitrary micrometer displacements resulting in noise floor of the voltage reference. Other solutions such as electronic filtration and bulk-micromachining instead of surface $\mu \mathrm{M}$ and the seesaw electrode which is damped by atmospheric pressure all could contribute to ameliorating rejection of voltage floor noise.
Position of the movable electrode is approximately constant during sinusoidal alterations in the AC current. Taking this into account, the electrostatic force in (3) can be substituted with the mean value of the electrostatic force. This brings about (5). Note that $\omega=2 \pi / T$ and in the steady state, around the pull-in point, $\ddot{x}$ and $\dot{x}$ approximate to zero.

$$
\begin{aligned}
\bar{F}_{E} & =\frac{2}{T} \int_{0}^{T / 2} \frac{I_{m}^{2} \cos ^{2}(\omega t)}{2 \epsilon A \omega^{2}} d t \\
& =\frac{1}{\pi} \int_{0}^{\pi} \frac{I_{m}^{2}(1+\cos (2 \alpha))}{4 \epsilon A \omega^{2}} d \alpha=\frac{I_{\mathrm{RMS}}^{2}}{2 \epsilon A \omega^{2} \pi}=k x .
\end{aligned}
$$

The electrostatic force which is applied on the movable plate can also get calculated by

$$
\bar{F}_{E}=\left|-\frac{\partial E_{V}}{\partial x}\right|=\frac{\epsilon A V_{\mathrm{RMS}}^{2}}{2(d-x)^{2}}=k x .
$$

In the above equation, $V_{\mathrm{RMS}}$ represents the Root Mean Square (RMS) of the AC voltage across the plates and $E_{V}$ is the electrostatic energy. Considering $x$ in terms of $I_{\mathrm{RMS}}$ from (5) and supplanting $x$ in (6) with the latter result yield (7) which asserts $V_{\mathrm{RMS}}$ in terms of $I_{\mathrm{RMS}}[23]$.

$$
V_{\mathrm{RMS}}=\frac{d}{\epsilon A \omega} I_{\mathrm{RMS}}-\frac{1}{2 k \epsilon^{2} A^{2} \omega^{3}} I_{\mathrm{RMS}}^{3} .
$$

Taking derivative of $V_{\text {RMS }}$ with respect to $I_{\text {RMS }}$ results in

$$
\frac{\partial V_{\mathrm{RMS}}}{\partial I_{\mathrm{RMS}}}=\frac{d}{\epsilon A \omega}-\frac{3}{2 k \epsilon^{2} A^{2} \omega^{3}} I_{\mathrm{RMS}}^{2} .
$$

We may attain the value of $I_{\mathrm{RMS}}^{\max }$ setting (8) to zero. It is equal to $\sqrt{2 k d \epsilon A \omega^{2} / 3}$. Supplanting $I_{\mathrm{RMS}}$ in (7) with $I_{\mathrm{RMS}}^{\max }$ yields $V_{\mathrm{RMS}}^{\max }$ called the reference or pull-in voltage:

$$
V_{\mathrm{RMS}}^{\max }=\sqrt{\frac{8 k d^{2}}{27 C_{0}}}=\sqrt{\frac{8 k d^{3}}{27 \epsilon A}} .
$$

The value of the corresponding $x$ is obtained by (10) considering (5) and (6) and also taking into account the attained maximum $V_{\mathrm{RMS}}^{\mathrm{max}}$ and its corresponding $I_{\mathrm{RMS}}^{\max }[23]$.

$$
x=\frac{d}{3}
$$

The major working point of MEMS tunable capacitors, in voltage reference source circuits, is the pull-in point. Consequently, small deviation in the input current around the pull-in point and its influence over the output voltage should be studied scrupulously. Using $I_{\mathrm{RMS}}+\Delta I$ instead of $I_{\mathrm{RMS}}$ in (7) results in

$$
\Delta V=\frac{d \Delta I}{\epsilon A \omega}-\frac{\left(\Delta I^{3}+3 I_{\mathrm{RMS}}^{\max ^{2}} \Delta I+3 I_{\mathrm{RMS}}^{\max } \Delta I^{2}\right)}{2 k \epsilon^{2} A^{2} \omega^{3}} .
$$


Equation (12) is achieved by dividing the above equation by $V_{\mathrm{RMS}}^{\max }$.

$$
\begin{aligned}
\frac{\Delta V_{\mathrm{RMS}}}{V_{\mathrm{RMS}}^{\max }} & =-\frac{3}{2}\left(\frac{\Delta I}{I_{\mathrm{RMS}}^{\max }}\right)^{2}-\frac{1}{2}\left(\frac{\Delta I}{I_{\mathrm{RMS}}^{\max }}\right)^{3} \\
& \approx-\frac{3}{2}\left(\frac{\Delta I}{I_{\mathrm{RMS}}^{\max }}\right)^{2} .
\end{aligned}
$$

Considering (12), it can be asserted that the voltage variations in the pull-in point are approximately proportional to the square of the current variations. Hence, small variations in the input current with respect to the pull-in current bring about a very small alteration in the pull-in voltage. This demonstrates that the tunable capacitor can be utilized as the major element in a voltage reference circuit. As mentioned before, assuming the actuation frequency to be much higher than the mechanical resonance frequency, the electrostatic force in (3) could be superseded with the mean value of the electrostatic force. This results in

$$
\bar{F}_{E}=\frac{I_{\mathrm{RMS}}^{2}}{2 \epsilon A \omega^{2} \pi}=u=m \ddot{x}(t)+b \dot{x}(t)+k x(t) .
$$

The describing equation of the plant can be modified as a nondimensional one by adopting $t^{\prime}$ as $t^{\prime}=\omega_{0} t$ and also dividing it by $m$ :

$$
\begin{aligned}
\omega_{0}^{2} & \frac{d^{2} x\left(t^{\prime} / \omega_{0}\right)}{d t^{\prime 2}}+\frac{b \omega_{0}}{m} \frac{d x\left(t^{\prime} / \omega_{0}\right)}{d t^{\prime}}+\frac{k}{m} x\left(\frac{t^{\prime}}{\omega_{0}}\right) \\
& =\frac{u\left(t^{\prime} / \omega_{0}\right)}{m} .
\end{aligned}
$$

Dividing the above equation by $\omega_{0}^{2}$ results in

$$
\begin{aligned}
\frac{u\left(t^{\prime} / \omega_{0}\right)}{m \omega_{0}^{2}}= & \frac{d^{2} x\left(t^{\prime} / \omega_{0}\right)}{d t^{\prime 2}}+\frac{b}{m \omega_{0}} \frac{d x\left(t^{\prime} / \omega_{0}\right)}{d t^{\prime}} \\
& +\frac{k}{m \omega_{0}^{2}} x\left(\frac{t^{\prime}}{\omega_{0}}\right) .
\end{aligned}
$$

Variables and parameters are defined as follows; $\ddot{x}^{\prime}=$ $d^{2} x\left(t^{\prime} / \omega_{0}\right) / d t^{\prime 2}, \dot{x}^{\prime}=d x\left(t^{\prime} / \omega_{0}\right) / d t^{\prime}, x^{\prime}=x\left(t^{\prime} / \omega_{0}\right), u^{\prime}=$ $u\left(t^{\prime} / \omega_{0}\right) / m \omega_{0}^{2}, b^{\prime}=b / m \omega_{0}$, and $k^{\prime}=k / m \omega_{0}^{2}$; the describing plant equation comes as

$$
\ddot{x}^{\prime}+b^{\prime} \dot{x}^{\prime}+k^{\prime} x^{\prime}=u^{\prime} \text {. }
$$

Omitting the prime symbol just to simplify representation of the above equation, the following dynamics come to describe the plant:

$$
\ddot{x}+b \dot{x}+k x=u,
$$

in which $b, k$, and $u$ are different from the ones in (13) or (14).

\section{Controller Design}

Considering the parametric uncertainties due to deficiencies in fabrication procedures and physical dimensions as $\Delta b$ and $\Delta k$ and remarking the disturbance imposed on the system from the input channel as $d$, the system dynamics are modified as follows:

$$
\ddot{x}+(b+\Delta b) \dot{x}+(k+\Delta k) x=u+d .
$$

All the parametric uncertainties and disturbance can be lumped by $l_{\text {un }}$ and the dynamics will consequently change into

$$
\ddot{x}+b \dot{x}+k x=u+l_{\text {un }} \text {. }
$$

Assuming parametric uncertainties and disturbance to be confined so that $\|\Delta b\| \leq \beta_{2},\|\Delta k\| \leq \beta_{1}$, and $\|d\| \leq \beta_{0}$, the lumped uncertainty will be bounded like this:

$$
\left\|l_{\text {un }}\right\| \leq \beta_{0}+\beta_{1}\|x\|+\beta_{2}\|\dot{x}\| .
$$

The reason is that

$$
\begin{aligned}
l_{\text {un }} & =d-\Delta b \dot{x}-\Delta k x \Longrightarrow\left\|l_{\text {un }}\right\| \\
& \leq\|d\|+\|\Delta b \dot{x}\|+\|\Delta k x\| \Longrightarrow\left\|l_{\text {un }}\right\| \\
& \leq \underbrace{\|d\|}_{\beta_{0}}+\underbrace{\|\Delta b\|}_{\beta_{2}}\|\dot{x}\|+\underbrace{\|\Delta k\|}_{\beta_{1}}\|x\| .
\end{aligned}
$$

We can also consider reference model describing equation as

$$
\ddot{x}_{m}=-a_{m} \dot{x}_{m}(t)-b_{m} x_{m}(t)+c_{m} r(t)
$$

to generate the desired trajectory. Note that the polynomial, $\sigma^{2}+a_{m} \sigma+b_{m}$, is a Hurwitz one in terms of $\sigma$ which guarantees the stability of the reference model. One would be able to tune the transient response specification adjusting $a_{m}$ and $b_{m}$. Unaffectedly, the tracking error is defined by

$$
e=x-x_{m} .
$$

The sliding surface is also considered as

$$
s(t)=\dot{e}+\lambda e
$$

in which $\lambda$ is a positive constant. Now, we would achieve timederivative of the sliding surface:

$$
\dot{s}(t)=\ddot{e}+\lambda \dot{e}=\ddot{x}-\ddot{x}_{m}+\lambda\left(\dot{x}-\dot{x}_{m}\right) .
$$

Remarking (19) and (22), the time-derivative of the sliding surface gets equal to

$$
\begin{aligned}
\dot{s}= & u+l_{\mathrm{un}}-W p+a_{m} \dot{x}(t)+b_{m} x_{m}(t)-c_{m} r(t) \\
& +\lambda\left(\dot{x}-\dot{x}_{m}\right),
\end{aligned}
$$

in which $W$ and $p$ are consecutively the regressor (trajectory) vector and the parameters' one. They are defined by the following terms:

$$
\begin{gathered}
W=\left[\begin{array}{ll}
\dot{x} & x
\end{array}\right] \\
p=\left[\begin{array}{ll}
b & k
\end{array}\right]^{T} .
\end{gathered}
$$


$W(x, \dot{x})$ is a function of trajectories. It is delineated since it is comprised of the accessible (measurable) states [23]. Considering (26), the following equivalent control law is adopted to zero variation of the sliding surface relative to time [28].

$$
\begin{aligned}
u_{\mathrm{eq}}= & -l_{\mathrm{un}}+W p-a_{m} \dot{x}(t)-b_{m} x_{m}(t)+c_{m} r(t) \\
& -\lambda\left(\dot{x}-\dot{x}_{m}\right)
\end{aligned}
$$

Regarding the unknown parametric uncertainties and disturbance, the control law is modified as

$$
\begin{aligned}
u= & u_{\text {lun }}+W \widehat{p}-a_{m} \dot{x}(t)-b_{m} x_{m}(t)+c_{m} r(t) \\
& -\lambda\left(\dot{x}-\dot{x}_{m}\right) .
\end{aligned}
$$

in which $u_{\text {lun }}$ is defined by

$$
u_{\text {lun }}=-\eta_{12} \operatorname{sgn}(s)
$$

and $\widehat{p}$ is the estimation of the unknown system parameters. In (31), $\eta_{12}$ is a positive constant which would be defined later and $s$ is the sliding surface in (24). It is noteworthy that the other terms in the adopted control law are defined or accessible ones. Now, we would simplify the time-derivative of the sliding surface regarding the control law in (30) and try to make the product of the regressor and parameters' estimation error vectors appear in the time-derivative of the sliding surface:

$$
\begin{aligned}
& \left.\begin{array}{c}
\dot{s}=u+l_{\text {un }}-W p+a_{m} \dot{x}(t)+b_{m} x_{m}(t)-c_{m} r(t)+\lambda\left(\dot{x}-\dot{x}_{m}\right) \\
u=u_{\text {lun }}+W \hat{p}-a_{\mathrm{m}} \dot{x}(t)-b_{m} x_{m}(t)+c_{m} r(t)-\lambda\left(\dot{x}-\dot{x}_{m}\right)
\end{array}\right\} \Longrightarrow \\
& \dot{s}=-\eta_{12} \operatorname{sgn}(s)+W \widehat{p}-a_{m} \dot{x}(t)-b_{m} x_{m}(t)+c_{m} r(t)-\lambda\left(\dot{x}-\dot{x}_{m}\right)+l_{\text {un }}-W p+a_{m} \dot{x}(t)+b_{m} x_{m}(t)-c_{m} r(t) \\
& +\lambda\left(\dot{x}-\dot{x}_{m}\right) \Longrightarrow \\
& \dot{s}=W \widetilde{p}+l_{\text {un }}-\eta_{12} \operatorname{sgn}(s) \text {. }
\end{aligned}
$$

In (32), $\widetilde{p}=\widehat{p}-p$ is the parameters' estimation error vector.

At this stage, we consider an augmented system of estimation and tracking errors so a Lyapunov candidate, as a function of the parameters' estimation error and the tracking error of the desired trajectory (sliding surface), is proposed. We may try to proffer the adaptive law (parameters' update law) so that the selected Lyapunov candidate function, $V(s, \widetilde{p})$, grows negative semidefinite. It brings about the boundedness of the tracking and parameters' estimation errors and consequently the stability of the system. The Lyapunov candidate function is proposed as

$$
V(s, \tilde{p})=\frac{1}{2} s^{2}+\frac{1}{2} \widetilde{p}^{T} \Gamma^{-1} \widetilde{p}
$$

in which $\Gamma_{2 \times 2}$ is a symmetric positive definite matrix. Differentiating the Lyapunov candidate relative to time leads to the following equation:

$$
\dot{V}=s \dot{s}+\dot{\tilde{p}}^{T} \Gamma^{-1} \widetilde{p}
$$

Regarding (32), $\dot{V}$ is obtained as

$$
\dot{V}=s\left(W \widetilde{p}+l_{\text {un }}-\eta_{12} \operatorname{sgn}(s)\right)+\dot{\tilde{p}}^{T} \Gamma^{-1} \widetilde{p} .
$$

It could be more simplified in the following:

$$
\begin{aligned}
& \dot{V}=s W \widetilde{p}+s l_{\text {un }}-\eta_{12} s \operatorname{sgn}(s)+\dot{\tilde{p}}^{T} \Gamma^{-1} \tilde{p} \Longrightarrow \\
& \dot{V}=s W \widetilde{p}+s l_{\text {un }}-\eta_{12}|s|+\dot{\tilde{p}}^{T} \Gamma^{-1} \tilde{p} \Longrightarrow \\
& \dot{V}=s l_{\text {un }}-\eta_{12}|s|+\underbrace{\dot{\tilde{p}}^{T} \Gamma^{-1} \tilde{p}+s W \widetilde{p}}_{\text {sign-indefinitet term }} .
\end{aligned}
$$

We may zero the above-mentioned sign-indefinite term trying to make the time-derivative of the Lyapunov function negative finally:

$$
\begin{aligned}
\dot{\tilde{p}}^{T} \Gamma^{-1} \widetilde{p}+s W \widetilde{p} & =0 \stackrel{\text { transpose }}{\longrightarrow} \widetilde{p}^{T} \Gamma^{-1} \dot{\tilde{p}}+\widetilde{p}^{T} W^{T} s=0 \Longrightarrow \\
\dot{\tilde{p}} & =-\Gamma W^{T} s .
\end{aligned}
$$

Note that $\Gamma$ is a symmetric matrix so $\left[\Gamma^{-1}\right]^{T}=\Gamma^{-1}$. The reason is illustrated below:

$$
\begin{aligned}
\Gamma^{-1} \Gamma & =I \stackrel{\text { transpose }}{\longrightarrow} \Gamma^{T}\left[\Gamma^{-1}\right]^{T}=I \Longrightarrow \\
\Gamma\left[\Gamma^{-1}\right]^{T} & =I \stackrel{\Gamma^{-1} \times}{\longrightarrow} \\
{\left[\Gamma^{-1}\right]^{T} } & =\Gamma^{-1} .
\end{aligned}
$$

Considering the environmental effects, the physical properties, and the working conditions of the device, it is assumed that the parameters vary very slowly; $\dot{\vec{p}}=\dot{\hat{p}}-\dot{p}=\dot{\hat{p}}$. Therefore, (37) turns into

$$
\dot{\hat{p}}=-\Gamma W^{T} s
$$

According to (36), (37), and (39), the time-derivative of the Lyapunov candidate equals

$$
\dot{V}=s l_{\text {un }}-\eta_{12}|s| \text {. }
$$

We may write the following inequality:

$$
\dot{V} \leq s l_{\text {un }}-\eta_{12}\|s\| \leq\|s\|\left\|l_{\text {un }}\right\|-\eta_{12}\|s\|,
$$


and consequently

$$
\dot{V} \leq-\|s\|\left(\eta_{12}-\left\|l_{\text {un }}\right\|\right) .
$$

If the positive constant $\eta$ is adopted so that $\eta=\eta_{12}-\left\|l_{\mathrm{un}}\right\|$, (42) would change into

$$
\dot{V} \leq-\|s\| \eta
$$

Equation (43) is the sliding condition which vouches for the trajectories to reach the sliding surface in finite time and remain there [28]. It also demonstrates that the Lyapunov function is negative semidefinite and the tracking and the parameters' estimation errors inevitably remain bounded. Therefore, $s(t) \in L_{\infty}$. Regarding (20), (32), and (27), we may conclude that $\dot{s}(t) \in L_{\infty}$. In other words,

$$
\left.\begin{array}{c}
\left\|l_{\text {un }}\right\| \leq \beta_{0}+\beta_{1}\|x\|+\beta_{2}\|\dot{x}\| \\
\dot{s}=W \widetilde{p}+l_{\text {un }}-\eta_{12} \operatorname{sgn}(s)
\end{array}\right\} \stackrel{s(t) \in L_{\infty}}{\longrightarrow} \dot{s}(t) \in L_{\infty} .
$$

On the other hand, $\dot{V}$ is negative semidefinite so $V$ is nonincreasing:

$$
V(t) \leq V(0)
$$

Integrating over the sliding condition, (43), we may write

$$
\int_{0}^{t}\|s(\tau)\| d \tau \leq \frac{1}{\eta}[V(0)-V(t)] .
$$

$V(0)$ is bounded, $V(t)$ is nonincreasing, and consequently inequality (46) turns into

$$
\int_{0}^{t}\|s(\tau)\| d \tau<\infty
$$

Therefore, $s(t) \in L_{1}$. Now, regarding the results, $s(t) \in L_{\infty}$, $\dot{s}(t) \in L_{\infty}$, and $s(t) \in L_{1}$, Barbalet's Lemma may be used to prove the zero-convergence of the composite error or the same sliding surface [28]:

$$
\left.\begin{array}{c}
s(t) \in L_{\infty}, \dot{s}(t) \in L_{\infty} \\
s(t) \in L_{1}
\end{array}\right\} \stackrel{\text { Barbalet's Lemma }}{\stackrel{\text { lima }}{s} s(t)=0 .}
$$

Ultimately, we have attained the goal of perfect tracking of the desired trajectory of the capacitive plate regarding (24) and (23); the plate would be placed in its right desired position in spite of the parametric uncertainties and the external disturbance.

The block diagram of the control scheme of the poleplacement state feedback controller is also displayed in Figure 3(a). The aim is controlling the movement of the capacitive plate so that it tracks the desired trajectory. Design of this controller is based on the convergence and stability of the tracking error [29]. The states are practically at the access for designing the closed loop control system. The capacitive plate should move in micrometers following the desired trajectory. Considering the desired trajectory and its
TABLE 1: Main parameters of tunable capacitor of the MEMS AC voltage reference.

\begin{tabular}{lccc}
\hline Parameter & Real value & Parameter & Real value \\
\hline$m$ & $1.11 \times 10^{-7} \mathrm{Kg}$ & $k$ & $2338 \mathrm{~N} / \mathrm{m}$ \\
$b$ & $0.01 \mathrm{Ns} / \mathrm{m}$ & $d$ & $2 \mu \mathrm{m}$ \\
\hline
\end{tabular}

first and second derivatives as $x_{m}, \dot{x}_{m}$, and $\ddot{x}_{m}$, the control input $\nu$ may be constructed by feedback of displacement and velocity of plate, $x$ and $\dot{x}$, as follows:

$$
\nu=\ddot{x}_{m}-2 \lambda \dot{\tilde{x}}-\lambda^{2} \tilde{x} .
$$

$\lambda>0$ is a tuning constant to adjust the response settling time and $\tilde{x}=x-x_{m}$. Utilizing the control input and the feedback of the states, the control law would be stated by the following equation:

$$
u=b \dot{x}+k x+\nu
$$

It is of great significance that the computed force described by (50) vouches for the stability of the error dynamics so that the tracking error $\tilde{x}$ tends to zero bringing about perfect tracking of the desired trajectory. If one substitutes $u$ in (50) for $u$ in (17), he would achieve stable error dynamics. Here is the verification of the statements:

$$
\begin{aligned}
\ddot{x}+b \dot{x}+k x & =\underbrace{b \dot{x}+k x+v}_{u} \Longrightarrow \\
\ddot{x} & =\ddot{x}_{m}-2 \lambda \dot{\tilde{x}}-\lambda^{2} \tilde{x} \Longrightarrow \\
\ddot{\tilde{x}}+2 \lambda \dot{\tilde{x}}+\lambda^{2} \tilde{x} & =0 .
\end{aligned}
$$

Equation (51) represents a Hurwitz characteristic equation so that $\tilde{x}$ tends to zero promisingly.

\section{Simulation Results}

The overall pole-placement state feedback controller system, displayed in Figure 3(a), is mainly comprised of 4 parts: plant dynamics (MEMS_AC_V_Ref or MEMS tunable cap.), reference model or desired trajectory generator, control input (ctrinput), and control law (ctrlaw). The pole-placement state feedback controller is simulated using parameter values of Table 1. The simulation results are seen in Figure 3(b). The red and blue curves are consecutively desired and real trajectories. The simulation is carried out under normal operating conditions. As it is observed, capacitive plate has tracked the desired trajectory. The point is that when the parameter uncertainty or the disturbance is considered in the plant dynamics, the overall control system loses its accuracy in tracking the desired trajectory or gets unstable. These claims are to be demonstrated through simulation in the following.

The overall control scheme of the adaptive sliding mode controller is seen in Figure 3(c). It is composed of 4 main blocks: the plant, the model reference, the control law, and the parameters' update law (estimator). The adaptive sliding mode controller is also simulated using the parameter values 


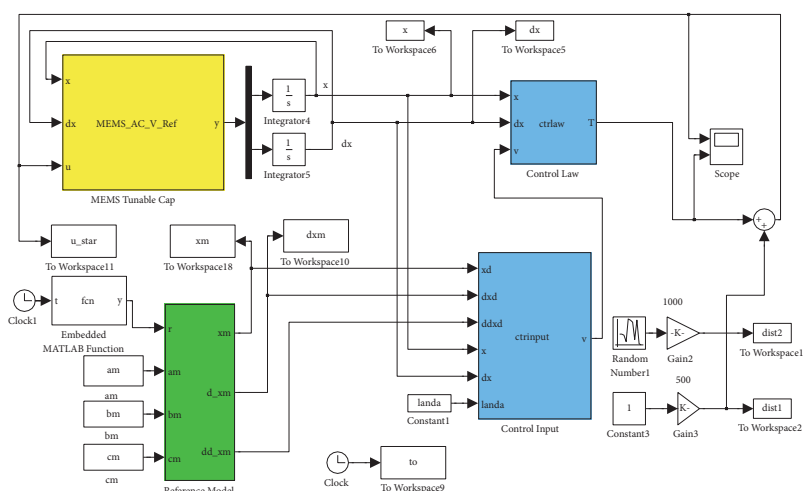

(a)

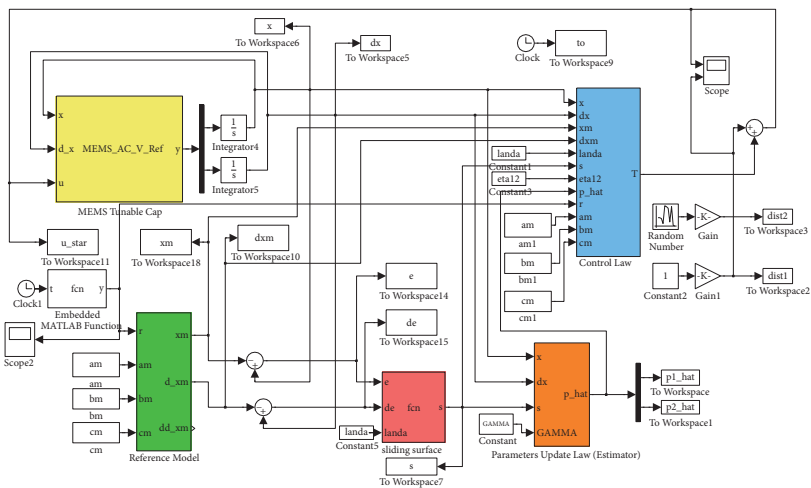

(c)

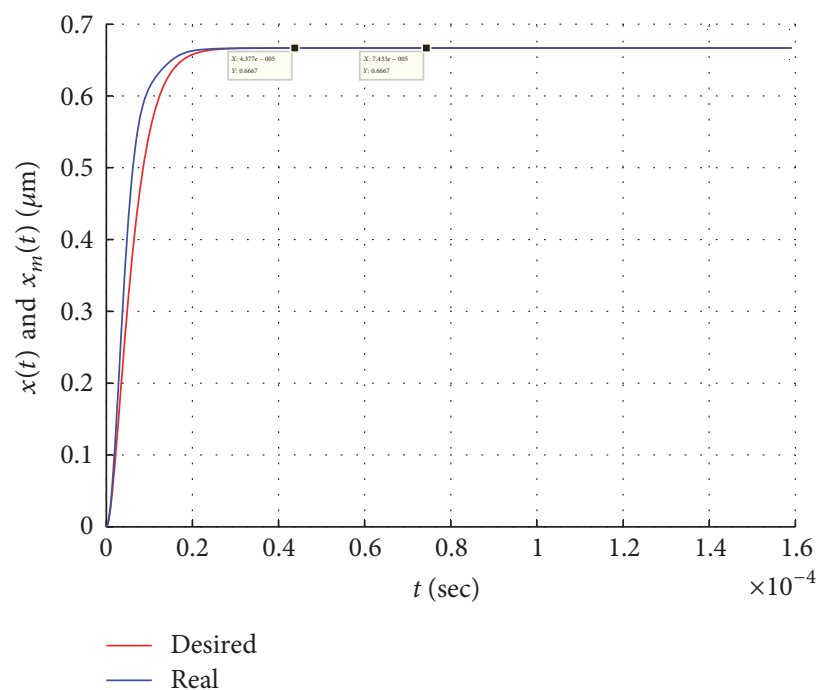

(b)

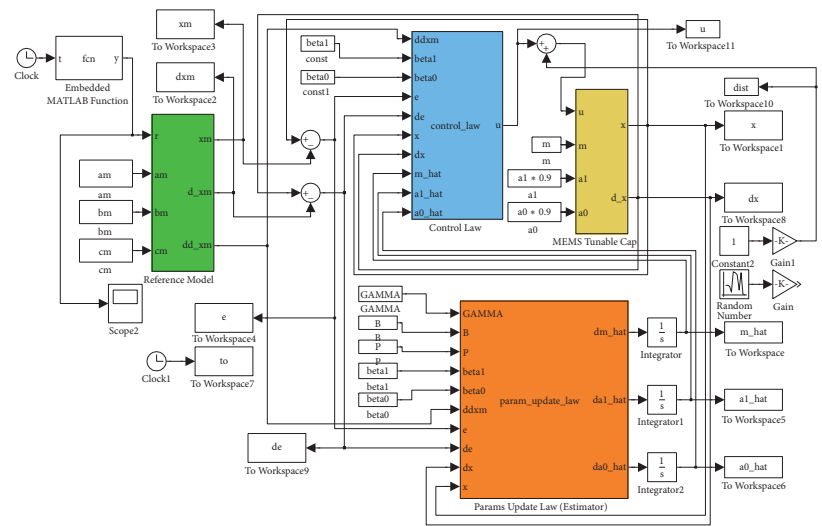

(d)

FIGURE 3: Simulation schemes of pole-placement state feedback controller, adaptive sliding mode, and conventional adaptive ones: (a) control scheme of the pole-placement state feedback controller; (b) tracking performance in the pole-placement state feedback controller under normal operating conditions; (c) adaptive sliding mode controller simulation scheme; (d) conventional adaptive controller simulation scheme [25].

in Table 1. In the simulations, a comparison between the adaptive sliding mode controller and the pole-placement and conventional adaptive ones has been illustrated. The controllers are simulated under the same operating conditions.

In the first comparative simulation, malfunctioning effect of parametric uncertainty is investigated. The overall adaptive sliding mode controller system is simulated with parameter values of ninety percent of the nominal ones. As it is observed, the adaptive controller has reached the goal of perfect tracking of desired position of the capacitive plate but the pole-placement controller has not. See Figures 4(a) and 4(b). The red and blue curves, in these figures, are correspondingly the defined tracking error and sliding surface on the first one and tracking error on the second. The estimated values of the plant parameters, damping and stiffness factors, remain bounded. The estimated value of stiffness converges to real one.

In the second comparative simulation, malfunctioning effect of slow parameter variation is theoretically investigated.
The overall adaptive sliding mode controller system is simulated with the parameter variation pattern of $k=k_{n}(1+$ $0.01 \sin (\pi t))$. As it is observed, the tracking performance has not collapsed seriously being compared with the tracking performance of the pole-placement state feedback controller. See Figure 4(c). The pole-placement controller is simulated considering the same parameter variation pattern. As it is seen in Figures 4(c) and 4(d), the tracking performance in the pole-placement controller has got ruined much more apparently and the goal of perfect tracking of desired position of the capacitive plate is out of reach. The capacitive plate is oscillating in an unacceptable range and it is not placed in its right desired position. The estimation errors remain bounded proving the adaptive system stability. The red and blue curves in Figures 4(c) and 4(d) have the same meaning correspondingly in Figures 4(a) and 4(b).

In the third comparative simulation, the two systems are simulated being subjected to the external constant disturbances which are imposed on the systems from the input 


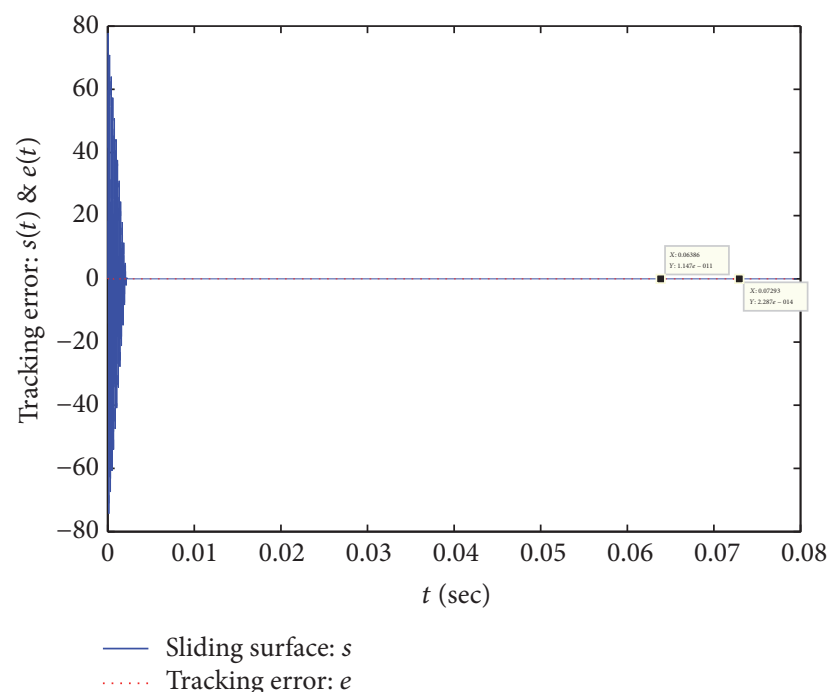

(a)

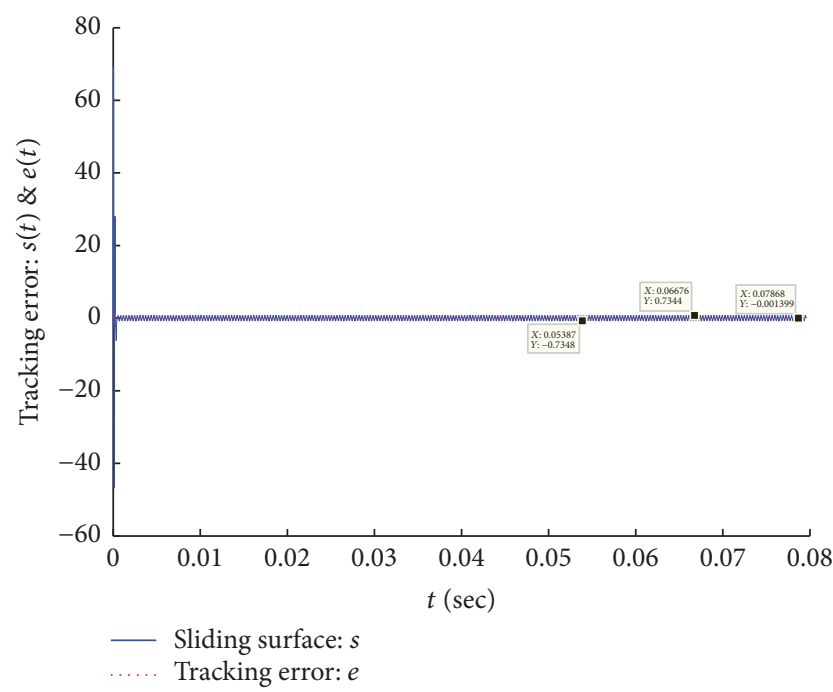

(c)

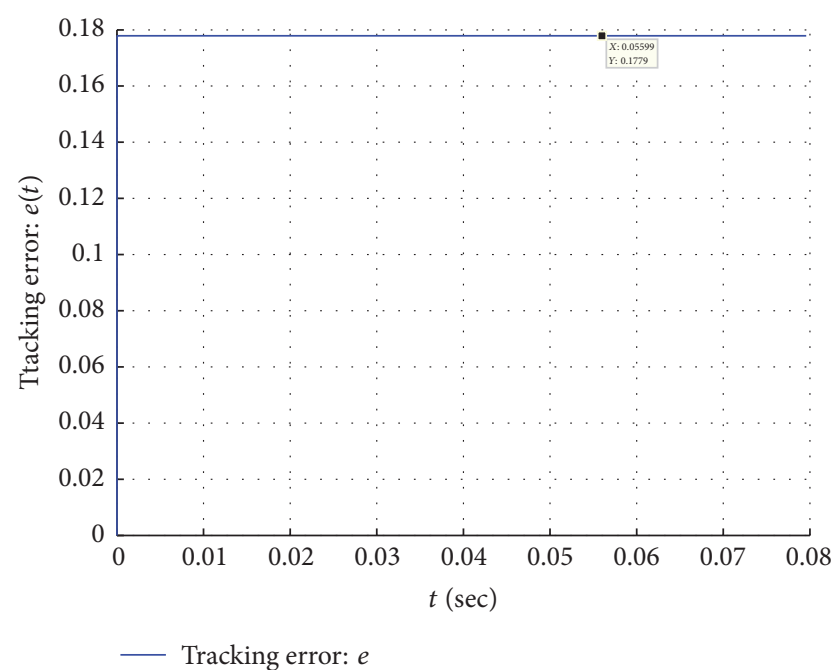

(b)

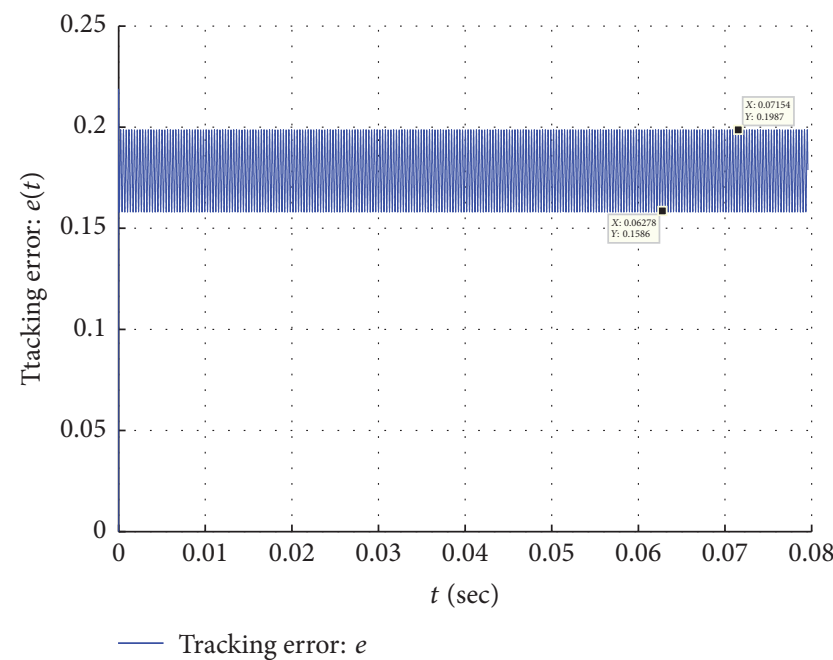

(d)

FIGURE 4: Tracking performance comparison between adaptive sliding mode controller and pole-placement state feedback one, both being susceptible to various malfunctioning effects: (a) tracking performance of adaptive sliding mode controller considering $10 \%$ divergence of the real values of stiffness and damping factors from the nominal ones; (b) tracking performance of pole-placement state feedback controller considering $10 \%$ divergence of the real values of stiffness and damping factors from the nominal ones; (c) tracking performance in adaptive sliding mode controller when it is susceptible to parameter uncertainty and variation; (d) tracking performance of pole-placement state feedback controller being susceptible to parameter uncertainty and variation.

channel. The tracking performance has got ruined in the presence of external constant disturbance in the pole-placement state feedback controller comparing Figures 5(a) and 5(b). The red and blue curves in these figures, on the upper graphs, come in affinity with the ones in Figures 4(c) and 4(d). On the lower graphs, they are consecutively disturbances and control efforts. As it can be observed, the pole-placement controller has lost its tracking performance being subjected to a much less disturbance relatively in comparison to the adaptive sliding mode controller. The tracking errors are seen in Figures 5(a) and 5(b). It is noteworthy that the promising simulation results demonstrate that the pole-placement controller gets unstable as the imposed disturbance grows higher. Again, the estimation errors remain bounded proving the stability of the adaptive sliding mode controller and the stiffness factor gets converged to the real value.

In the fourth comparative simulation, the systems are simulated being prone to the external random disturbances. They are imposed on the system from the input channel. It would be concluded that the tracking performance has collapsed intensively in the presence of external random disturbance in the pole-placement state feedback controller; an intuitive comparison can be made between Figures 6(a) and 6(b). Again, the red and blue curves are the familiar plots 


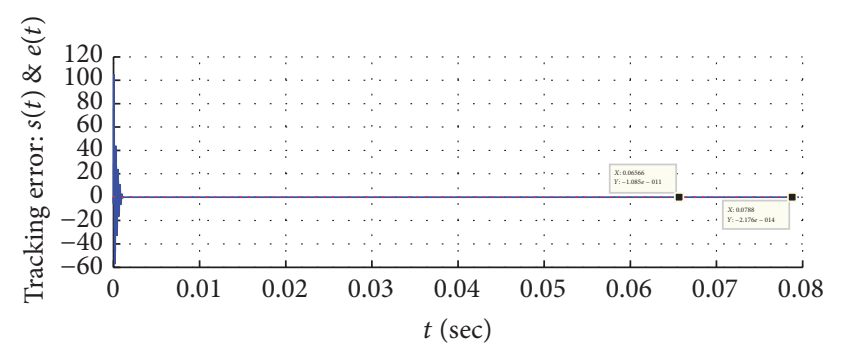

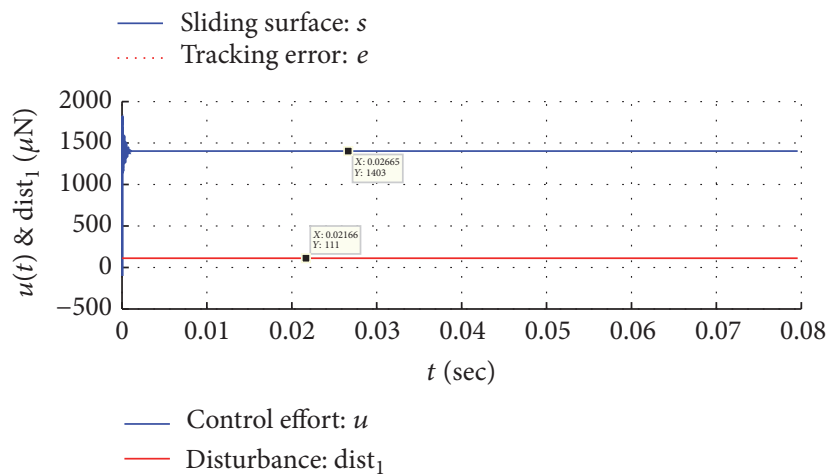

(a)

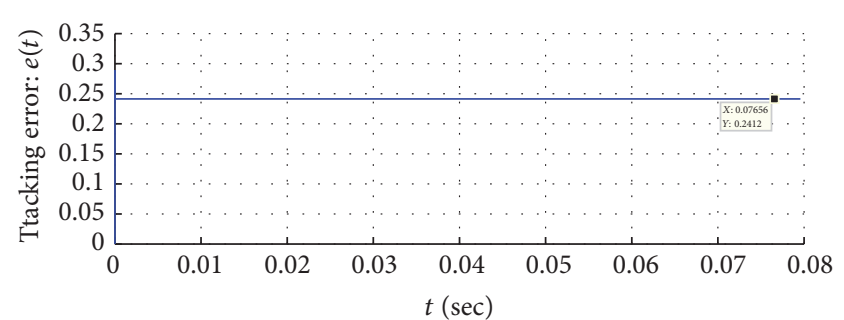

— Tracking error: $e$

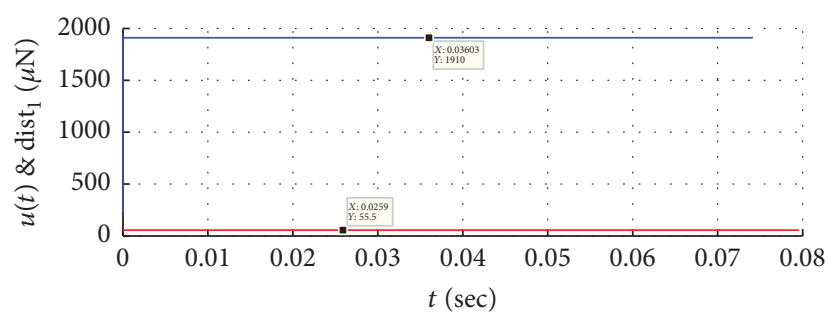

Control effort: $u$

- Disturbance: dist ${ }_{1}$

(b)

FIGURE 5: Tracking performance comparison between adaptive sliding mode controller and pole-placement state feedback one, both being susceptible to various malfunctioning effects: (a) tracking performance of adaptive sliding mode controller being prone to parameter uncertainty and constant disturbance; (b) tracking performance of pole-placement state feedback controller being prone to parameter uncertainty and constant disturbance.
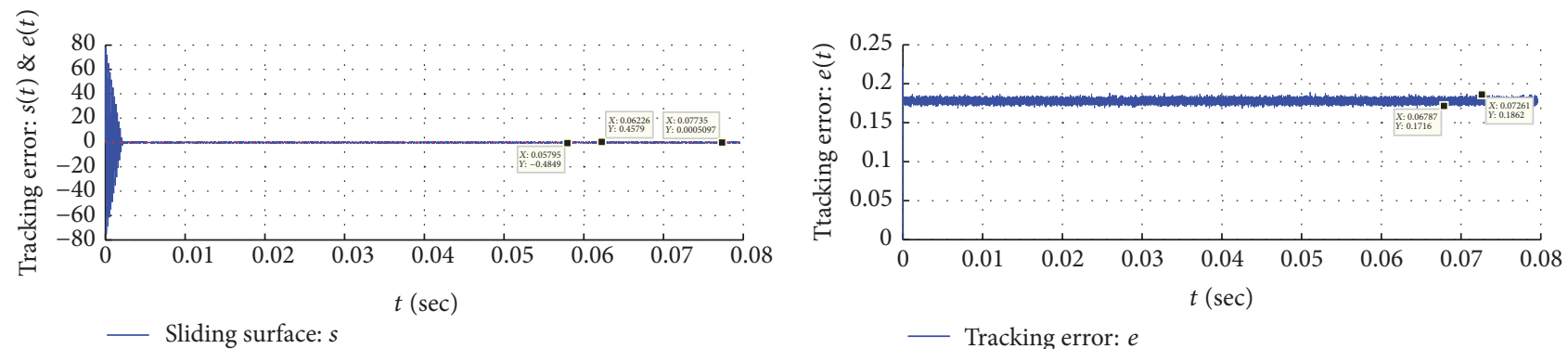

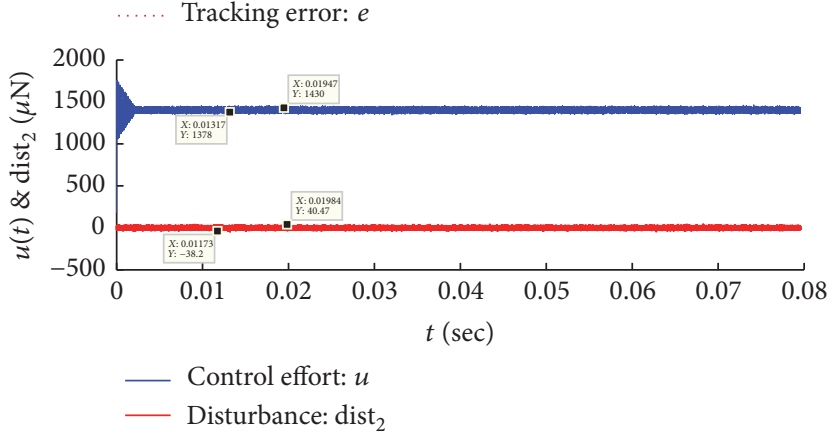

(a)

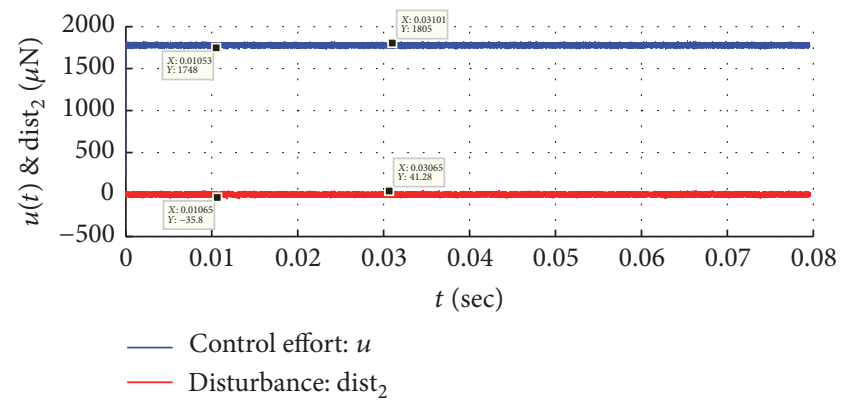

(b)

FIGURE 6: Tracking performance comparison between adaptive sliding mode controller and pole-placement state feedback one, both being susceptible to various malfunctioning effects: (a) tracking performance of the adaptive sliding mode controller in presence of parameter uncertainty and random disturbance; (b) tracking performance of the pole-placement state feedback one in presence of parameter uncertainty and random disturbance. 

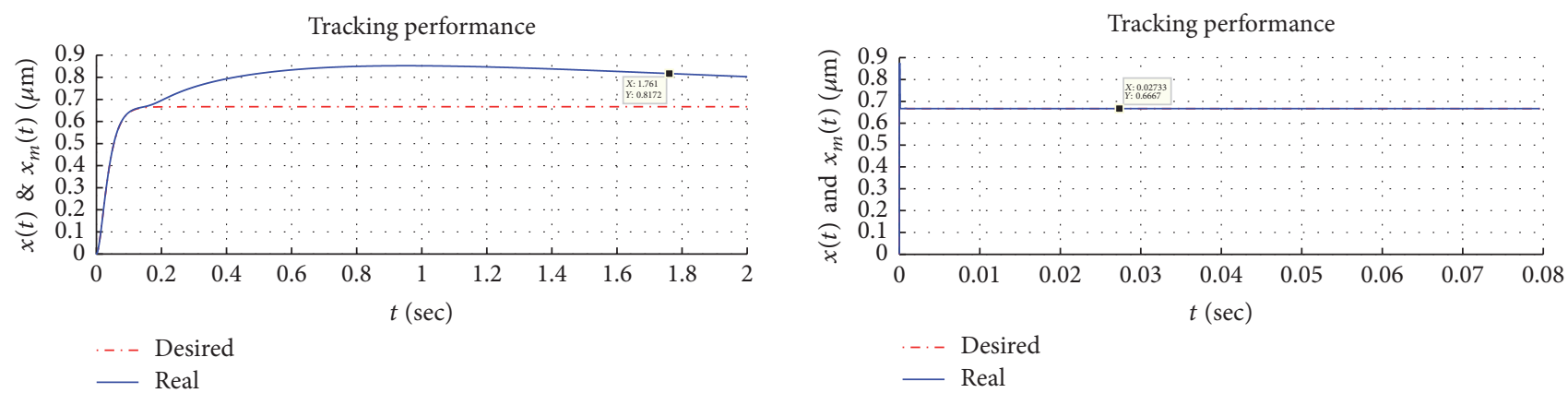

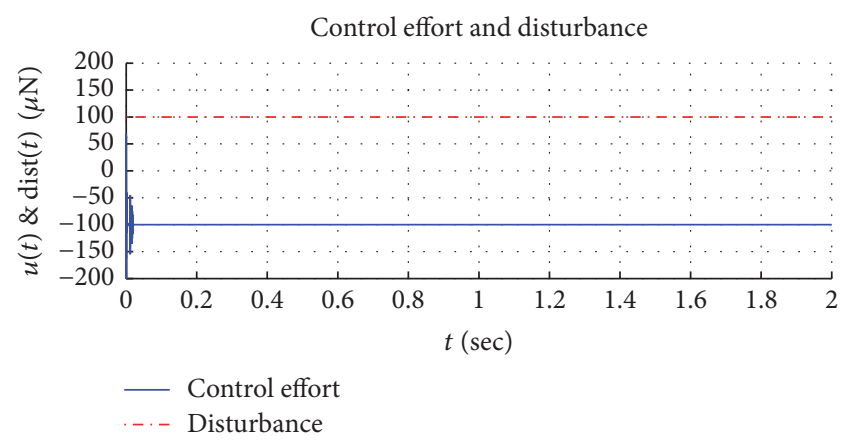

(a)

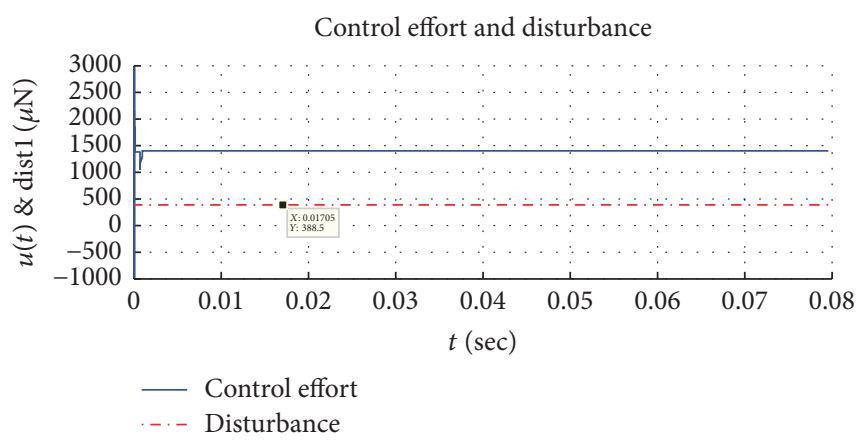

(b)

FIGURE 7: Tracking performance comparison between adaptive sliding mode controller and the conventional one: (a) tracking performance in conventional adaptive controller. It is vulnerable to parameter uncertainty and constant disturbance; (b) tracking performance in adaptive sliding mode controller. It is also prone to parameter uncertainty and constant disturbance.

presenting the tracking error, the sliding surface, disturbance, and control effort. The pole-placement controller is deprived of perfect tracking investigating the tracking error displayed in Figure 6(b). The simulation results pledge the stability of the adaptive sliding mode controller bringing about boundedness of estimation errors.

In the fifth and final comparative simulation, the robustness of the adaptive sliding mode controller and that of the conventional adaptive one against external disturbance are also compared. The conventional adaptive controller scheme [25] is displayed in Figure 3(d). As it can be seen in Figure 7(a), this controller is incapable of perfect tracking of desired position of the capacitive plate. The simulation is carried out using parameter values of Table 1 . The disturbance imposed on the adaptive sliding mode controller is considered greater than the disturbance imposed on the conventional adaptive one. The red curves are correspondingly the desired trajectory and the disturbance imposed on the system and the blue curves are the real trajectories and the control efforts, in Figures 7(a) and 7(b). The switching control effort, $u_{\text {lun }}=-\eta_{12} \operatorname{sgn}(s)$ in (31), is the main cause of reinforced disturbance rejection mechanism. Decreasing chattering effect, other so-called "smooth transient switching functions" like $u_{\text {lun }}=-\eta_{12} \operatorname{sat}(s)$ or $u_{\text {lun }}=-\eta_{12} \tanh (s)$, which is used in the simulations, could be used in the proposed adaptive sliding mode controller [28].

The last important issue which is worthwhile being notified here is that, from a mechanical engineering vantage point, the physical system should keep up with the control effort vacillation and its consequent frequency impact on the mechanical behaviour. According to the modal analysis of the tunable capacitor carried out by Abaqus software, the socalled "starting mode" or the first mode is 53392 cycles/s. See Figures 8(a) and 8(b). As it is depicted in Figure 8(c), the control effort signal in frequency domain does not comprise the trouble-making frequency of the first mode and beyond, inhibiting vulnerability of deformation. It does not make any difference if the disturbance is considered or both disturbance and parameter uncertainty are taken into account.

\section{Conclusion}

MEMS tunable capacitors can be modeled as the major elements of the AC voltage reference sources. The movable plate of capacitor should be maneuvered towards the pull-in point obtaining the desired output voltage. The uncertainties in the dynamics of this device make it difficult to accomplish the aim of perfect tracking utilizing the simple pole-placement state feedback controller or other classic ones. Meticulous endeavors are devoted to fabrication procedures to obtain the requested physical characteristics of the tunable capacitor. Nonetheless, some parametric uncertainties ineluctably transpire in some parameters of the device like stiffness and damping coefficients. The suggested adaptive sliding mode controller is competent of flawless tracking of desiderated position trajectory of the movable plate in defiance of the parametric uncertainties and also external disturbance. As a result, a well-regulated output voltage is given off by the voltage reference source. The overall system has the benefit of disturbance rejection being compared with the conventional 

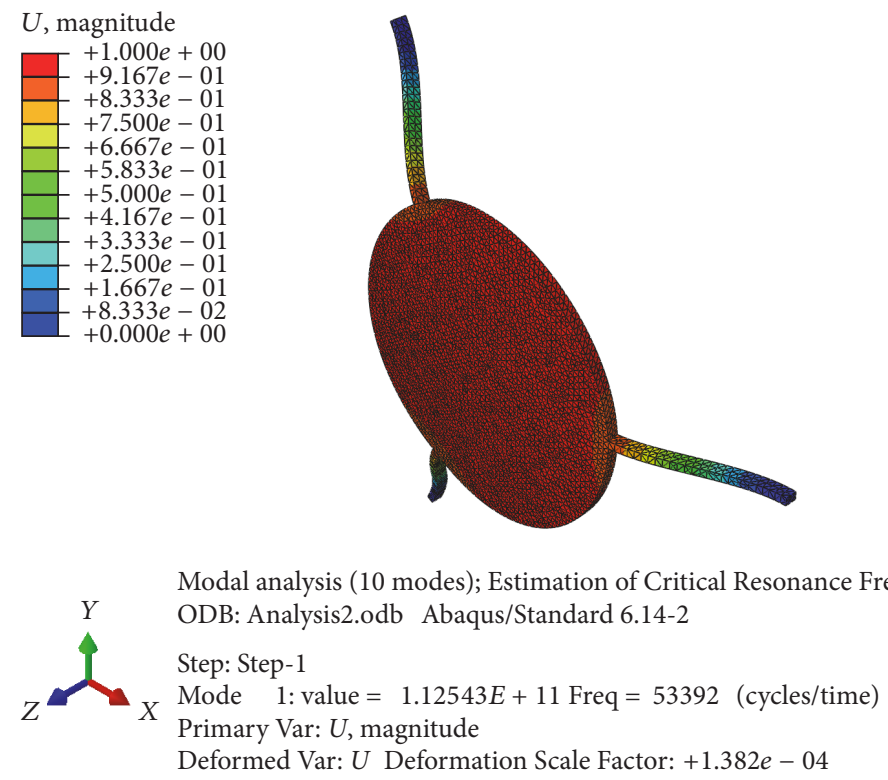

(a)
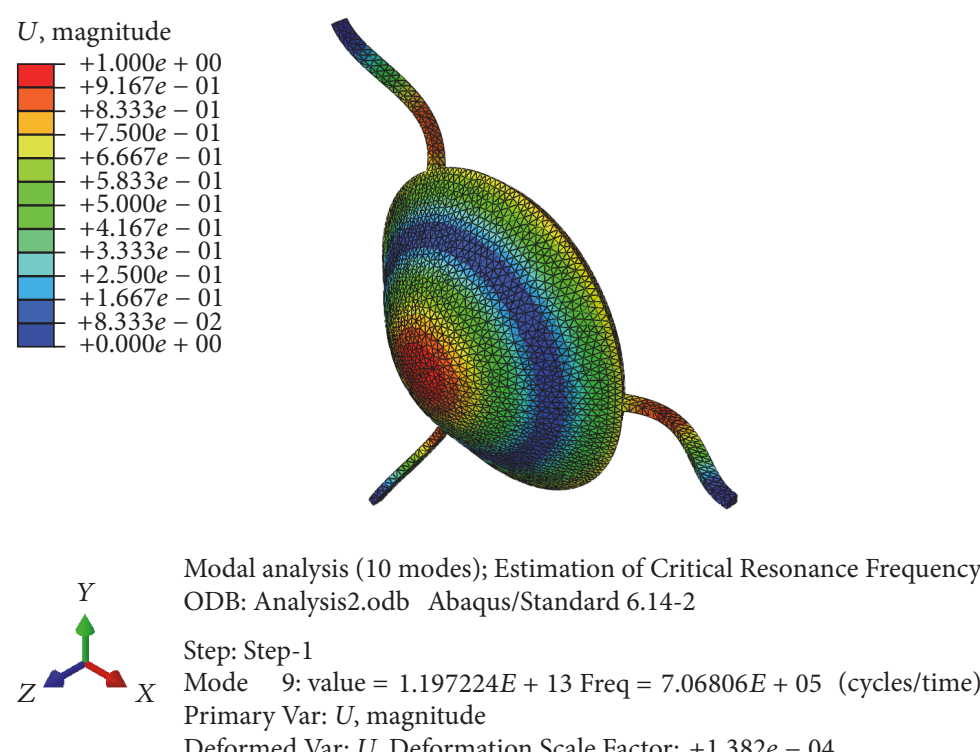

(b)
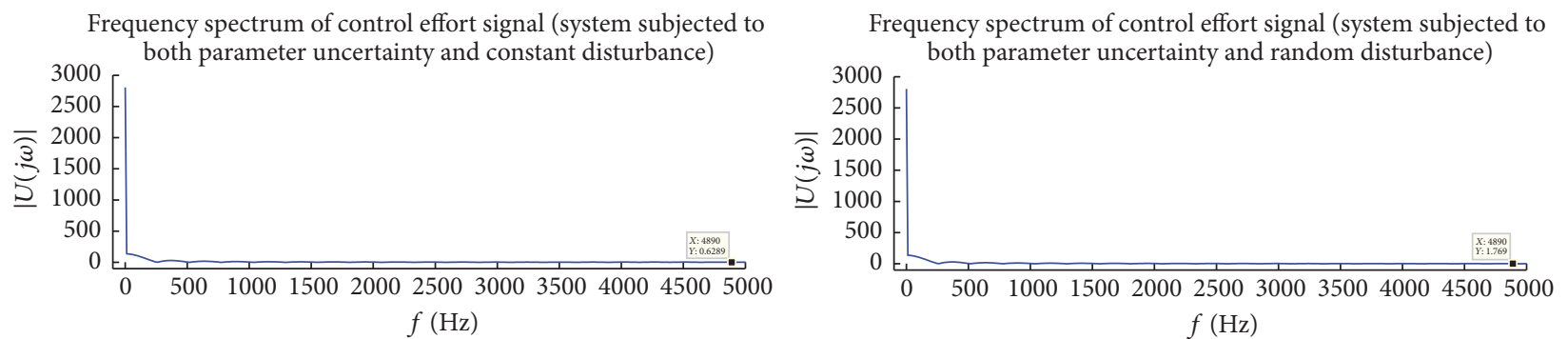

(c)

FIGURE 8: Mechanical analysis: ((a) and (b)) deformation of top plate and its displacement in modal analysis carried out in 10 modes; deformation in mode 1 and in mode 9; (c) spectrum of control effort signal in the adaptive sliding mode controller. 
adaptive controller; it also shows remarkable robustness against the exacerbating disturbance imposed on the system and the slow parameter variation.

\section{Future Work}

Leaving no stone in micrometer dimensions unturned, it should be notified that the damping coefficient may function as the displacement in a nonlinear manner. According to [30], the following equation comes to describe squeezed film damping.

$$
b=\frac{64 \sigma P_{a} A}{\pi^{6} d} \sum_{m, n \text { odd }} \frac{m^{2}+c^{2} n^{2}}{(m n)^{2}\left[\left(m^{2}+c^{2} n^{2}\right)^{2}\right]+\sigma^{2} / \pi^{4}},
$$

where $P_{a}$ is the ambient pressure, $m$ and $n$ are odd integers, and $c=w / l$ and $A=w l$ for a plate with width $w$ and length l. Plate separation is $d$ and squeeze number $\sigma$ works as a function of frequency $\omega$ :

$$
\sigma=\frac{12 \mu_{\mathrm{eff}} w^{2}}{P_{a} d^{2}} \omega
$$

in which $\mu_{\text {eff }}$ is the gas effective viscosity. For larger frequencies, it seems that damping coefficient functions as the frequency nonlinearly:

$$
b=\frac{1}{\omega} \sum_{j} \alpha_{j}
$$

In this way, damping factor keeps constant but whether this approximating conclusion holds to be true needs verification research to be carried out. Another issue which requires one's prospective attention is parameter identification working alongside the adaptive controller to estimate stiffness and damping factors for catalogue purposes. Furthermore, design and tuning a fuzzy adaptive sliding mode controller to bring more robustness to the controller and investigation of its possible landmark effect over rejection of disturbance and reduction of voltage noise floor could be deemed as other upcoming research field.

\section{Disclosure}

Ali Mehrnezhad is currently a Ph.D. student in Electronics area in Electrical Engineering: Micro-Scaled Biomedical Device Lab, Division of Electrical and Computer Engineering, Louisiana State University, Baton Rouge, LA 70803, USA.

\section{Conflicts of Interest}

The authors declare that there are no conflicts of interest regarding the publication of this paper.

\section{Acknowledgments}

The authors wish to acknowledge the support of MEMS/ NEMS Dynamics Research Lab in AUT.

\section{References}

[1] M. Suhonen, H. Seppa, A. Oja, M. Heinila, and I. Nakki, "AC and DC voltage standards based on silicon micromechanics," in Proceedings of the Conference on Precision Electromagnetic: Measurements, pp. 23-24.

[2] A. Karkkainen, N. Pesonen, M. Suhonen et al., "AC voltage reference based on a capacitive micromechanical component," in Proceedings of the 2004 Conference on Precision Electromagnetic Measurements, pp. 119-120, June 2004.

[3] A. Kärkkäinen, P. Pekko, J. Dekker et al., "Stable SOI micromachined electrostatic AC voltage reference," Microsystem Technologies, vol. 12, no. 1-2, pp. 169-172, 2005.

[4] F. Blard, A. Bounouh, D. Bélières, and H. Camon, "Very high stability achievement in MEMS based AC voltage references," in Proceedings of the 24th IEEE International Conference on Micro Electro Mechanical Systems, MEMS '11, pp. 656-659, January 2011.

[5] A. Bounouh, H. Camon, D. Bélières, F. Blard, and F. Ziadé, "MEMS AC voltage reference for miniaturized instrumentation and metrology," Computer Standards and Interfaces, vol. 33, no. 2, pp. 159-164, 2011.

[6] A. Bounouh, H. Camon, and D. Bélières, "MEMS based AC voltage references with very high stability," in Proceedings of the 2012 Conference on Precision Electromagnetic Measurements, CPEM '12, pp. 552-553, July 2012.

[7] A. Bounouh, H. Camon, and D. Bélières Denis, "Wideband high stability MEMS-based AC voltage references," IEEE Transactions on Instrumentation and Measurement, vol. 62, no. 6, pp. 1646-1651, 2013.

[8] A. Kärkkäinen, A. Oja, J. Kyynäräinen, H. Kuisma, and H. Seppä, "Stability of electrostatic actuation of MEMS," in Proceedings of the 20th Nordic Semiconductor Meeting, NSM20, pp. 193-194, August 2003.

[9] J. Fei and C. Batur, "A novel adaptive sliding mode control with application to MEMS gyroscope," ISA Transactions, vol. 48, no. 1, pp. 73-78, 2009.

[10] C. Batur, T. Sreeramreddy, and Q. Khasawneh, "Sliding mode control of a simulated MEMS gyroscope," ISA Transactions, vol. 45, no. 1, pp. 99-108, 2006.

[11] N. Sadeghzadeh-Nokhodberiz and J. Poshtan, "Loosely coupled fusion of camera and inertial sensors for distributed error compensation in strapdown inertial navigation system," Transactions of the Institute of Measurement and Control, vol. 38, no. 11, pp. 1283-1297, 2016.

[12] B. Parsi, M. Bahrami, A. M. Esfahani, and B. S. Sany, "Calibration verification of a low-cost method for MEMS accelerometers," Transactions of the Institute of Measurement and Control, vol. 36, no. 5, pp. 579-587, 2014.

[13] Q. Xu, P.-K. Wong, M. Jia, M. Rakotondrabe, and L. Zhang, "Advanced control in micro-/nanosystems," Journal of Control Science and Engineering, vol. 2012, Article ID 827479, 2 pages, 2012.

[14] M. Meenakshi and M. S. Bhat, "Real-time fixed-order lateral H2 controller for micro air vehicle," Journal of Control Science and Engineering, vol. 1, 15 pages, 2011.

[15] J. Fei, "Robust adaptive vibration tracking control for a microelectro-mechanical systems vibratory gyroscope with bound estimation," IET Control Theory and Applications, vol. 4, no. 6, pp. 1019-1026, 2010.

[16] J. Fei, M. Xin, and W. Juan, "Adaptive fuzzy sliding mode control using adaptive sliding gain for MEMS gyroscope," Transactions 
of the Institute of Measurement and Control, vol. 35, no. 4, pp. 551-558, 2013.

[17] W. Juan and J. Fei, "Adaptive fuzzy approach for non-linearity compensation in MEMS gyroscope," Transactions of the Institute of Measurement and Control, vol. 35, no. 8, pp. 1008-1015, 2013.

[18] D. Wu and J. Fei, "Adaptive neural sliding control of MEMS gyroscope with robust feedback compensator," Transactions of the Institute of Measurement and Control, vol. 38, no. 4, pp. 414424, 2015.

[19] A. Modirrousta, M. Shokrian Zeini, and T. Binazadeh, "Nonlinear optimal fuzzy control synthesis for robust output tracking of uncertain micro-electro-mechanical systems," Transactions of the Institute of Measurement and Control, vol. 39, no. 8, pp. 1146-1160, 2016.

[20] D. Derawi, N. D. Salim, H. Zamzuri, M. A. A. Rahman, and K. Nonami, "Robust attitude control design for a low-cost hexarotor micro aerial vehicle (MAV)," Transactions of the Institute of Measurement and Control, 2016.

[21] S. Choura, N. Aouni, and S. El-Borgi, "On the control of vibratory MEMS gyroscopes," Smart Structures and Systems, vol. 6, no. 7, pp. 793-810, 2010.

[22] F. A. Hassane, A. F. Payam, and M. Fathipour, "Design of a smart MEMS accelerometer using nonlinear control principles," Smart Structures and Systems, vol. 6, no. 1, pp. 1-16, 2010.

[23] A. Mehrnezhad, A. A. Suratgar, S. Khatami, and S. Sobhiyeh, "A mathematical dynamic model for static and dynamic behaviours of MEMS-based AC voltage reference source," in Proceedings of the 2013 21st Iranian Conference on Electrical Engineering, ICEE '13, May 2013.

[24] J. Fei, Advanced Control Design of MEMS Vibratory Gyroscope, Nova Science Publishers, New York, NY, USA, 2012.

[25] E. Ranjbar, A. Mehrnezhad, A. A. Suratgar, and S. Khatami, "Adaptive control of MEMS-based AC voltage reference source," in Proceedings of the 22nd Iranian Conference on Electrical Engineering, ICEE '14, pp. 1336-1341, May 2014.

[26] A. Kärkkäinen and A. Maija, Dissertation for the degree of Doctor of Philosophy to be presented with due permission of the Department of Electrical and Communications Engineering for public examination and debate in the Large Seminar Hall of Micronova at Helsinki University of Technology, VTT Technical Research Centre of Finland, Finland, 2006.

[27] L. Castaner, J. Pons, R. Nadal-Guardia, and A. Rodriguez, "Analysis of the extended operation range of electrostatic actuators by current-pulse drive," Sensors and Actuators, A: Physical, vol. 90, no. 3, pp. 181-190, 2001.

[28] J. E. Slotine and W. Li, Applied Nonlinear Control, Prentice Hall, Upper Saddle River, New Jersey, NJ, USA, 1991.

[29] W. L. Brogan, Modern Control Theory, Prentice-Hall, Upper Saddle River, New Jersey, NJ, USA, 3rd edition, 1991.

[30] C. Acar and A. Shkel, MEMS Vibratory Gyroscopes; Structural Approaches to Improve Robustness, Springer, New York, NY, USA, 2008. 


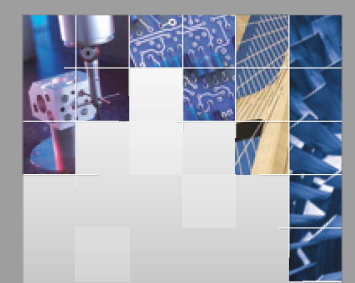

\section{Enfincering}
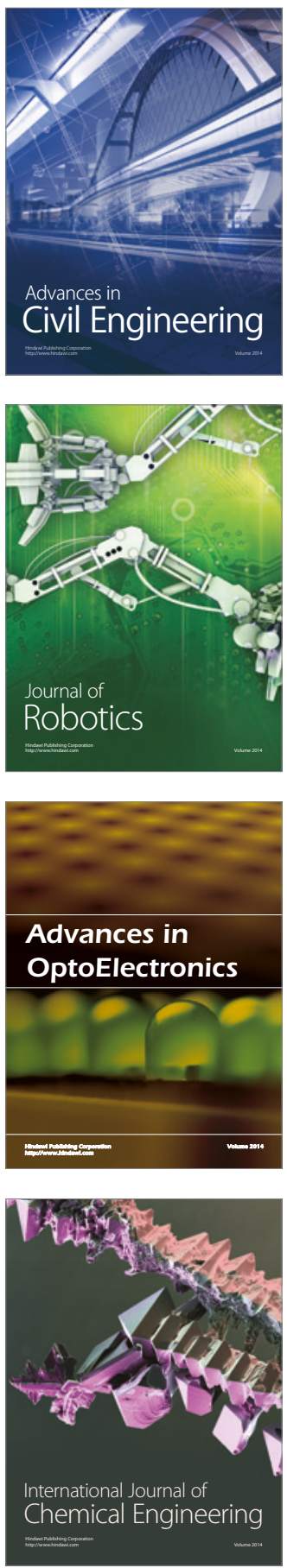

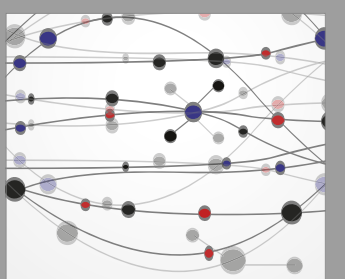

The Scientific World Journal

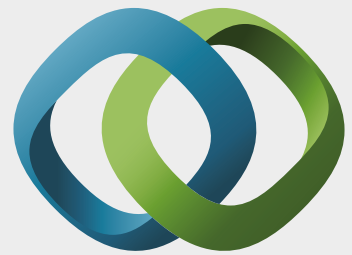

\section{Hindawi}

Submit your manuscripts at

https://www.hindawi.com
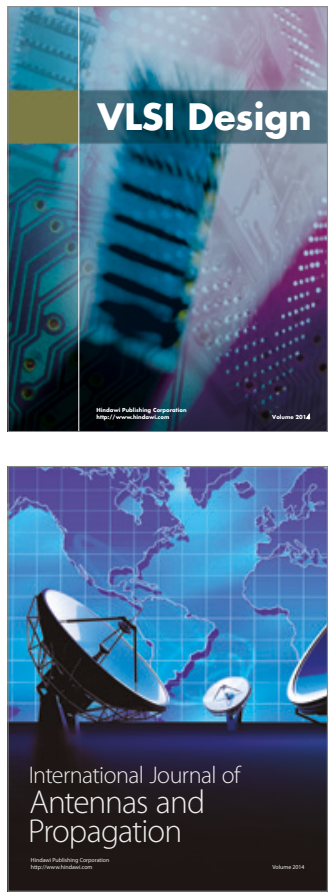

\section{Rotating}

Machinery
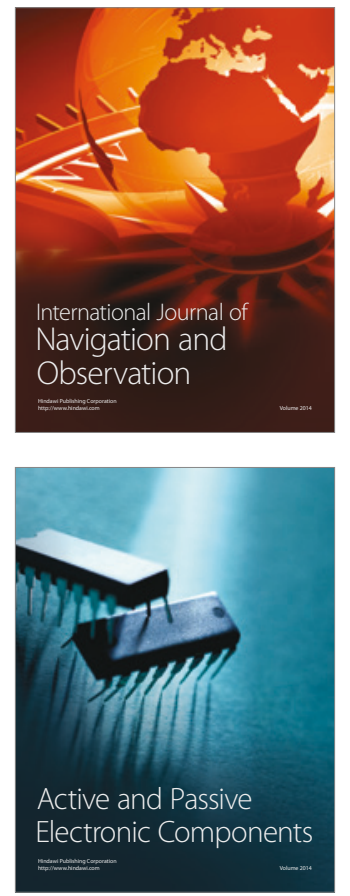
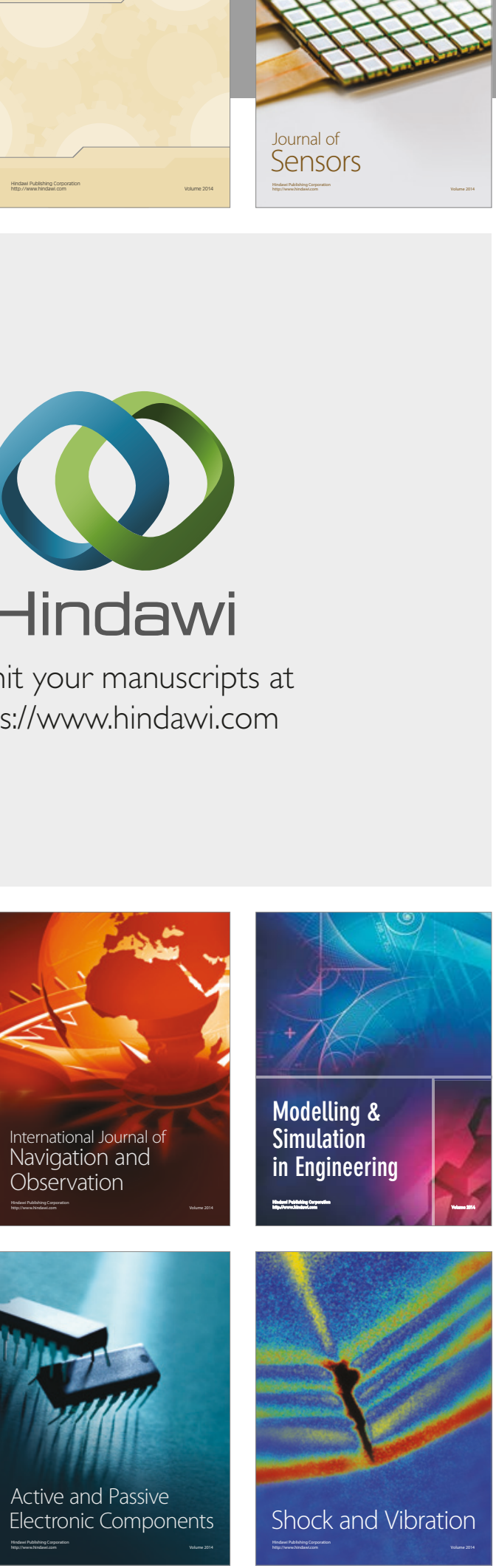
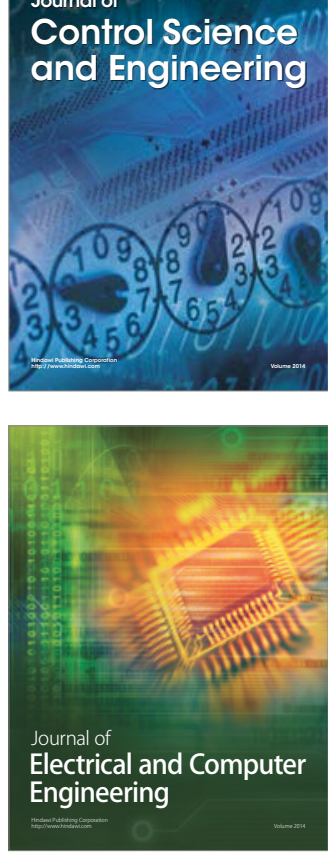

Distributed

Journal of

Control Science

and Engineering
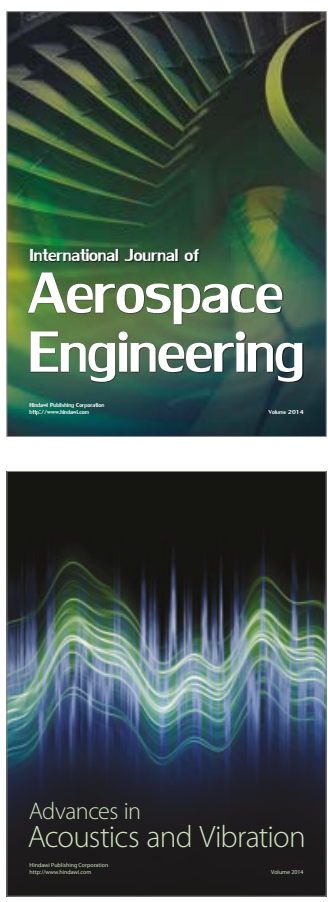

Sensor Networks 